\title{
Reflective Phase Properties of two-dimensional Photonic Crystal within The Bandgaps Based on Surface Structures Regulation
}

\author{
ZhonghuaLuo, Zhixiang Tang \\ Department of Information science and Engineering, Hunan University, Changsha, 10532, China
}

Keywords: PWE method, two-dimensional photonic crystal, FDTD Method, surface structure regulation, photonic bandgap, reflection phase.

\begin{abstract}
Using the plane wave expansion method to determine the band gap of two dimensional photonic crystal, and then through the finite difference time domain method with relevant software and numerical calculation we study the influence of two-dimensional photonic crystal structure of photonic band gap on the reflective phase. The research results show that in the photonic band gap, reflectance is constant 1 . We analyzed TM mode influence of the bandgap on the reflective phase and found that we can realize modulation of the reflective phase by surface structures regulation of two-dimensional photonic crystals.
\end{abstract}

\section{Introduction}

In photonic crystal materials[1-2], the two-dimensional photonic crystals is more flexible than the one-dimensional in application, and is easier than the three-dimensional photonic crystals on the preparation. Therefore, the two-dimensional photonic crystal is one of the most studied photonic crystal structure.Amplitude, frequency and phase are used to characterize the three basic physical quantities of the light.However, in the research of photonic crystals, a lot of research focused on the transmission or reflection amplitude and frequency[3-5],the phase of research are relatively few[6-7].Photonic conduction band can realize the above-mentioned three quantity control by the changes of refractive index, but the impedance mismatch may cause a modulation for broadband signal spectrum[8]. Despite photonic bandgap lost control ability of amplitudebecause of total reflection, but the reflection phase based on photonic band gap provides a new way of implementation for phase regulation.

The most important features and applications of photonic crystals were found in photonic band gap.Such as ZhongshanUniversityput forward a method to design broadband phase retardersby using the reflection of a two-dimensional photonic crystal band gap phase characteristics[9]. But based on the photonic band gap how to regulate the reflection phase has not been studied. This paper studies the reflection phase based on surface modulation. On the one hand, surface modulation widely used photons crystal surface impedance and increase the rate of transmission, suggests that there is a corresponding control ability[10]; on the other hand, face modulation for photonic crystals is easy to operation and practice.

\section{Theory}

The 2D finite difference time domain method(FDTD) is adopted to calculate complex reflection coefficient and obtain reflection phase shift. On one hand, FDTD is very effective for calculation of complex reflection. On the other hand, there is more freedom to set unit cell structure in FDTD, which allows us to adjust structural in a broad region to optimize structures. A complex reflection coefficient is defined as $R=E_{r} / E_{\text {in }}$, where $E_{r}$ is the field of reflected light, and $E_{\text {in }}$ is the field of the incident light. The complex reflection coefficient is given as follow:

$$
\boldsymbol{R}=|\boldsymbol{R}| e^{i \varphi}(1)
$$

Where $|R|$ is the reflection coefficient and $\varphi$ is the phase shift in the reflected light.

First, we use the plane wave expansion method for two-dimensional photonic crystal band, determining the first band gap frequency range. Then we select the appropriate center frequency 
$\omega_{0}$ for the Gaussian pulse, using relevant software EastWAVE(using the finite difference time domain method) and MATLAB numerical to calculate phase of the reflected light.

\section{Phase changes of two-dimensional photonic crystal bandgap}

We first consider a 2D square array of dielectric rods in background material. The rod has the dielectric constant ea $=12.96$ and the radius $\mathrm{r}=0.25 \mathrm{a}$, where $\mathrm{a}$ is the lattice constant. The background material of dielectric constant is $e b=2.6569$. In this paper, we set $a=268.515 \mathrm{~nm}$. Its structure is given as shown in figure1(a). Using the plane wave expansion method, energy band diagram is plotted, as shown in figure1(b). The red part is medium and the blue part is cylindrical.

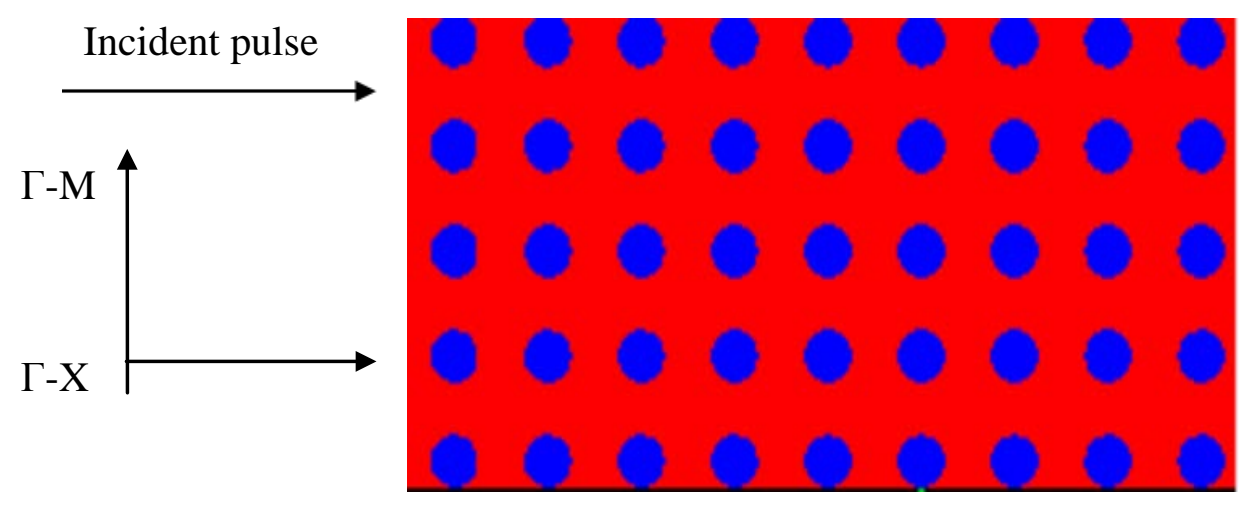

Fig.1(a): two-dimensional photonic crystals structure

In this article, we use the finite difference time domain method to simulate the photonic crystal structure as fig 1(a). The direction of the light incident from air is along $\Gamma-\mathrm{X}$, normal to the surface of the PC. In the vertical propagation plane direction, using periodic boundary conditions show that the direction of the structure of the photonic crystal is an infinite thick; in the direction of propagation , we use perfectly matched layer (PML) absorbing boundary condition.We select center frequency $\omega_{0}=284.7 \mathrm{THz}$, and a half wide time $\mathrm{t}=600 *$ dtgaussian pulse source for the incident $(\mathrm{dt}$ for simulation unit of time).

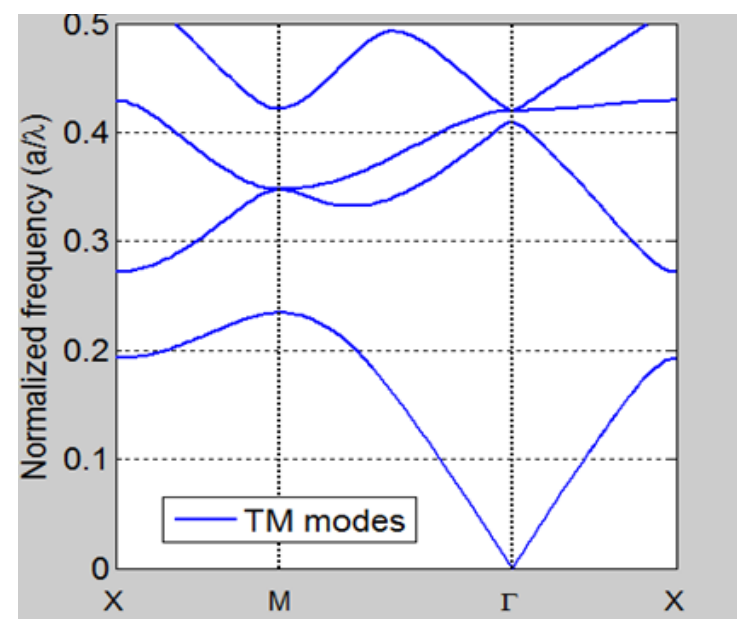

Fig.1(b): TM modes of the photonic band structure diagram

The complex reflection coefficient is defined as $R=|R| e^{i \varphi}$, and the reflection coefficient model in the photonic crystal band gap range of the frequency is constant of 1 . The reflection phasepin the center frequency $\omega_{0}$ by Taylor series expansion as follow:

$$
\varphi(\omega)=\left.\sum_{m=0} \frac{1}{m !} \frac{d^{m} \varphi}{d \omega^{m}}\right|_{\omega=\omega_{0}}\left(\omega-\omega_{0}\right)^{m}
$$

We can get the first order and second order phase near the center frequency of $\omega_{0}$ forbidden band of the rate of change. 
Within a scope of the original cell of the photonic crystal truncation, truncation distance $\mathrm{s}$ respectively $0,0.25 a, 0.5 a, 0.75 a$, the reflected light reflectivity is shown in figure 2.The figure 2 shows that photonic band gap (reflectivity is 1) in figure 1 (b) corresponds to the photonic bandgap ofbasically the same when $s=0$ is not truncated, which show that this method is feasible.

The figure shows within a scope of the original cell of the photonic crystal truncation, has no effect on the forbidden band. The first order and two order phase rate of change near the center frequency $\omega_{0}$ of the forbidden band is obtained from formula (2), as shown in fig 3 and fig 4, respectively.

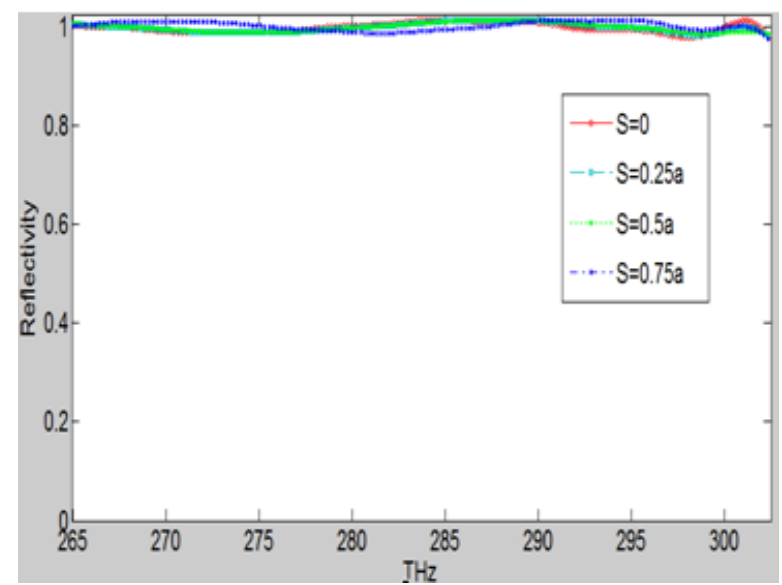

Fig.2: different corresponding truncation distance of reflectivity

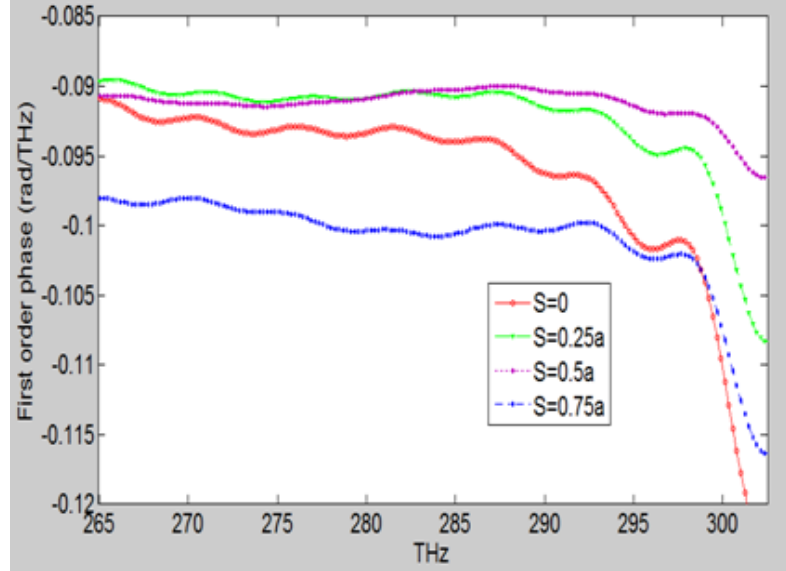

Fig.3: different truncated distance corresponding to the first-order phase rate of change

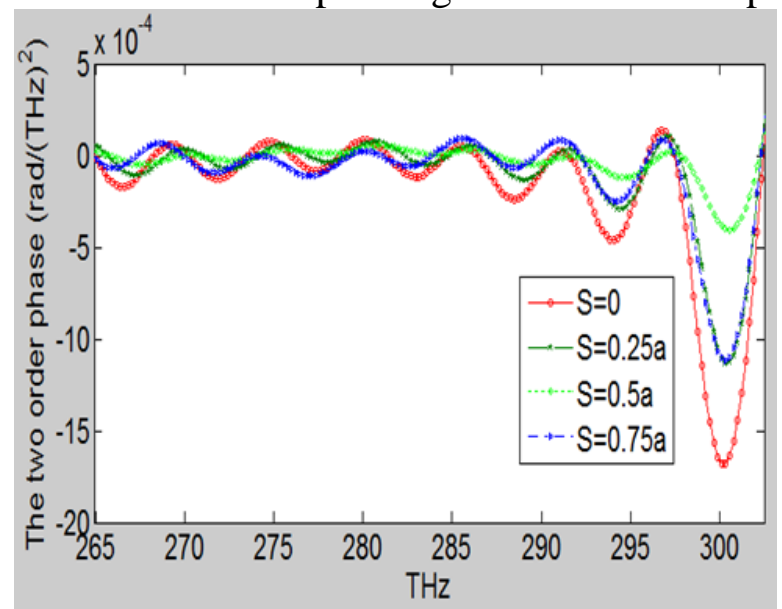

Fig.4: different truncated corresponding to the second order phase rate of change of distance Within the scope of an unit cell of the photonic crystal truncation, the first order phase rate of change is small between the $s=0$ to $s=0.5 a$, but with the increase of truncation distance, when $\mathrm{s}=0.75 \mathrm{a}$, there will be relatively obvious increase and both of them are negative as shown in figure 3 . The second order phase rate of change is very little on the frequency of the band gap range for $265 \mathrm{THz}-295 \mathrm{THz}$, but this is changing fairly obvious near $300 \mathrm{THz}$ with the increase of truncation 
distance within the scope of an unit cell.

\section{Conclusions}

In summary, using FDTD, the first order and second order phase rate of change in the first band gap of 2D photonic crystal based on TM model have been investigated with the increase of truncation distance in the scope of an unit cell. The results of numerical calculation and analysis can be seen that different end truncation distance has no effect on the band gap range. In the forbidden band frequency range $265 \mathrm{THz}-295 \mathrm{THz}$, the first order and second order phase rate of change has little change, but this is changing fairly obvious near $300 \mathrm{THz}$. Through the interesting findings (different truncated distance), we can choose the frequency with which we are interested in. On the one hand, in the forbidden band frequency range, we can choose that the first order and second order phase almost don't change such as at $265 \mathrm{THz}-295 \mathrm{THz}$. On the other hand, we can obtain relatively large phase rate of change such as near $300 \mathrm{THz}$.

\section{References}

[1] E. Yablonovitch, Inhibited spontaneous emission in solid-state physics and electronics.Phys. Rev. Lett. 58, 2059(1987).

[2]John D. Joannopoulos, Photonic Crystals. Princeton University Press, 1995.

[3] A. V. Andreev, A. V .Balakin, I. A.Ozheredov, and A. P. Shkurinov, Compression of femtosecond laser pulses in thin one-dimensional photonic crystals. Physical Review E, volume 63, 016602(2000).

[4] D. Chigrin, S. Enoch, C.Sotomayor Torres, and G. Tayeb, Self-guiding in two-dimensional photonic crystals. Opt. Express 11,1203-1211(2003).

[5] X. Yu and S. Fan, Bends and splitters for self-collimated beams in photonic crystals. Appl. Phys. Lett. 83,3251-3253(2003).

[6]Y. H. Chen, Unusual transmission bands of one-dimensional photonic crystals containing single-negative materials. Opt. Express 17, 20333-20341(2009).

[7]Y. H. Chen, W. X. Wang, Z.H. Yong, Y. J. Zhang, and Z. F. Chen, Experimental investigation of photonic band gap in one-dimensional photonic. Phys. Lett. A 376, 1396-1400(2012).

[8]T. J. Karle, Y. J. Chai, I. H. White, and T. F. Krauss, Observation of pulse compression in photonic crystal coupled cavity waveguides, Journal of Lightwave Technology, VOL,22, NO, 2, 2004.

[9]Q. F. Dai, S. Lan, L. J. Wu, and H. Z. Wang, Phase properties of reflected light in photonic band gap.Journal of Applied Physics 107, 093108(2010).

[10]Felix J. Lawrence, C. Martijn de Sterke, Lindsay C. Botten, R. C. McPhedran, and Kokou B. Dossou, Modeling photonic crystal interfaces and stacks:impedance-based approaches. Advances in Optics and Photonics 5, 385-455(2013). 